Martin Graversen*, Sönke Detlefsen, Jon Asmussen, Bassam Mahdi, Claus Fristrup, Per Pfeiffer and Michael Bau Mortensen

\title{
Treatment of peritoneal carcinomatosis with Pressurized IntraPeritoneal Aerosol Chemotherapy - PIPAC-OPC2
}

https://doi.org/10.1515/pap-2018-0108

Received April 18, 2018; accepted April 21, 2018;

previously published online June 9, 2018

\section{Abstract}

Background: Peritoneal carcinomatosis (PC) is a common endpoint in both gastrointestinal and non-gastrointestinal cancers, and PC is treated as other systemic metastases - unfortunately with disappointing results and considerable side-effects. Pressurized IntraPeritoneal Aerosol Chemotherapy (PIPAC) is a new method of applying traditional chemotherapy, and preliminary data indicate that PIPAC is safe, able to stabilize or improve quality of life, and can induce an objectively measurable reduction in disease burden in PC.

Methods: PIPAC-OPC2 is a prospectively controlled Phase II, single center, one-arm, open-label clinical trial investigating the treatment effect of PIPAC in patients with histological or cytological proven PC from gastrointestinal, ovarian or primary peritoneal cancer. Eligible patients will receive PIPAC in series of three using a combination of doxorubicin $\left(1.5 \mathrm{mg} / \mathrm{m}^{2}\right)$ and cisplatin

*Corresponding author: Martin Graversen, Odense PIPAC Center (OPC) and Odense Pancreas Center (OPAC) HPB and Upper GI Section, Department of Surgery, Odense University Hospital, Odense, Denmark, E-mail: martin.graversen@rsyd.dk

Sönke Detlefsen, Odense PIPAC Center (OPC) and Odense Pancreas Center (OPAC), Department of Pathology, Odense University Hospital, Odense, Denmark, E-mail: sonke.detlefsen@rsyd.dk Jon Asmussen: E-mail: jon.asmussen@rsyd.dk, Bassam Mahdi: E-mail: bassam.mahdi@rsyd.dk, Odense PIPAC Center (OPC) and Odense Pancreas Center (OPAC), Department of Radiology, Odense University Hospital, Odense, Denmark

Claus Fristrup, Odense PIPAC Center (OPC) and Odense Pancreas Center (OPAC) HPB and Upper GI Section, Department of Surgery, Odense University Hospital, Odense, Denmark, E-mail: claus.wilki. fristrup@rsyd.dk

Per Pfeiffer, Odense PIPAC Center (OPC) and Odense Pancreas Center (OPAC), Department of Oncology, Odense University Hospital, Odense, Denmark, E-mail: per.pfeiffer@rsyd.dk

Michael Bau Mortensen, Odense PIPAC Center (OPC) and Odense Pancreas Center (OPAC) HPB and Upper GI Section, Department of Surgery, Odense University Hospital, Odense, Denmark,

E-mail: michael.bau.mortensen@rsyd.dk $\left(7.5 \mathrm{mg} / \mathrm{m}^{2}\right)$ for non-colorectal cancer patients (PIPAC $\mathrm{C} / \mathrm{D})$, and oxaliplatin $\left(92 \mathrm{mg} / \mathrm{m}^{2}\right)$ in patients with $\mathrm{PC}$ of colorectal origin (PIPAC OX). Patients are monitored by: (1) repeated measurements of the Peritoneal Regression Grading Score (PRGS) in biopsies obtained from metal clips marked areas, (2) Quality-of-Life (QoL) questionnaires, (3) Magnetic Resonance Imaging (MRI) and (4) Prognostic Nutritional Index (PNI). Adverse events and surgical complications will be recorded according to the 30 days definition.

Results: The primary outcome of PIPAC-OPC2 is to evaluate if PIPAC can induce major or complete response (PRGS 1 or 2) within a series of three PIPAC procedures. Secondarily this study investigates changes in QoL and MRI as a staging and response evaluation tool. The secondary outcomes will be used to create a model that may predict which of the patients will benefit from PIPAC treatment.

Conclusions: It is expected that PIPAC directed therapy can induce major or complete response in $50 \%$ of patients with PC of colorectal origin and in $30 \%$ of patients with PC of non-colorectal origin - and at the same time stabilize or even improve quality of life. This trial may provide data regarding the utility of MRI as a staging and response evaluation tool in patients with PC. Trial registration: The study is registered with ClinicalTrials.gov Identifier NCT03287375 and the European Clinical Trials Database (EudraCT) number 2016-003394-18.

Keywords: MRI, peritoneal carcinomatosis, peritoneal regression grading score (PRGS), PIPAC, quality of life, response evaluation.

\section{Introduction}

Peritoneal carcinomatosis (PC) represents end stage disease in many types of cancer (e.g. gastric, pancreatic, liver, colo-rectal, ovarian) and, if left untreated, the majority of patients with PC will die from their disease 
within 6 months [1]. Platinum-based systemic chemotherapy (SC) may prolong survival in selected patients, but poor performance status and low response rates have led to nihilistic treatment strategies in these patients. However, patients with PC who are in good condition and with a remaining life expectancy of more than a few months, may still have an unmet need for additional treatment in order to be able to perform with a high quality of life for as long as possible. These patients have often tried several lines of SC with disappointing results, and alternative and more effective treatment strategies are needed.

The installation of chemotherapeutic agents within the peritoneal cavity would seem a simple and effective treatment of PC - at least from a theoretical point of view. Unfortunately, the effect of intraperitoneal chemotherapy on $\mathrm{PC}$ is disappointing. Like with $\mathrm{SC}$, the poor response rates may be explained by the poor penetration of the active chemical substances into the PC plaques [2]. Combining cytoreductive surgery (CRS) with hyperthermic intraperitoneal chemotherapy (HIPEC) has gained interest in recent years, but this approach can only be used in highly selected patients and carries a significant risk of complications and side effects [1]. Thus, a minimally invasive and safe delivery of relevant chemotherapeutics in concentrations that allow it to work efficiently on the PC plaques would be an ideal tool for a new treatment strategy in these patients.

\section{Pressurized IntraPeritoneal Aerosol Chemotherapy (PIPAC)}

PIPAC has been established as a new drug delivery system that may be used in patients with PC. PIPAC is a safe procedure $[3,4]$ with a very low adverse events profile [5-7], and although no Phase III studies have been published so far, the preliminary clinical experience seems promising in patients with PC from several different types of primary cancers [8]. A recent international survey study showed that the PIPAC procedure, the time between procedures and the chemotherapy protocols were similar in most centers [9]. However, the methods used to evaluate disease burden before, during and after PIPAC-directed treatment (i.e. treatment response) were more inhomogeneous. Traditional staging tools (e.g. CT and PET-CT) have been validated for status at the time of surgery, not for the progression/regression of $\mathrm{PC}$ at repeated laparoscopic procedures. With these limitations in mind, Sugarbaker's Peritoneal Cancer Index (PCI) is used as a surrogate marker for treatment response, whereas repeated biopsies for histologic analysis are more seldom used [9].

\section{Peritoneal Regression Grading Score (PRGS) and peritoneal lavage fluid examination}

In 2016 an international expert pathology group suggested the use of a Peritoneal Regression Grading Score (PRGS) to evaluate biopsies during PIPAC-directed treatment [10], but data comparing baseline PRGS with PRGS in biopsies retrieved at the third PIPAC procedure are still lacking. When looking at the peritoneal fluid, free intraperitoneal tumor cells (FITC) detected during peritoneal lavage seem associated with a poor prognosis in various cancer types (e.g. gastric cancer) [11]. By combining conventional cytology (with immunocytochemistry, if needed), protein analysis and reverse transcriptase polymerase chain reaction (RT-PCR) for carcinoembryonic antigen (CEA) mRNA, the conversion rate from FITC positive to FITC negative patients may be assessed, and thus provide an additional marker for the effect of PIPAC directed therapy.

\section{MRI}

In a recent study of patients with ovarian cancer, diffusion-weighted MRI, PET-CT and CT were compared with perioperative findings at diagnostic laparoscopy. When comparing the size and location of PC, MRI had an accuracy of 0.94 , compared to 0.75 and 0.71 for CT and PET-CT [12]. When comparing PCI scores between preoperative combined diffusion-weighted and gadolinium-enhanced MRI and PCI scores obtained during laparotomy the sensitivity of MRI was 0.88 and the specificity 0.74 [13]. In a recent, relatively small retrospective study, the same group presented a sensitivity of 0.95 and a specificity of 0.70 when comparing PCI scores from combined diffusion weighted and gadolinium enhanced MRI with PCI scores during laparotomy [14]. Based on these results, diffusionweighted and gadolinium enhanced MRI seems to be able to predict the PCI score, but it is unknown if it can be used as a tool in the response evaluation.

\section{Prognostic Nutritional Index}

PC is perceived as end-stage disease and some of the referred patients are beyond therapeutic reach. To 
ensure optimal patient selection, the need for an initial low-cost analysis without side effects seems obvious. Based on the total lymphocyte count and serum albumin level, the Prognostic Nutritional Index (PNI) is correlated to postoperative complications and long term prognosis in patients with gastric cancer $(10 \times$ serum albumin value $(\mathrm{g} / \mathrm{dl})+0.005 \times$ lymphocyte count in peripheral blood) $[15,16]$. In a study of 248 gastric cancer patients, a low PNI correlated with more advanced tumor size, nodal involvement and venous and lymphatic invasion. By dividing the patients into two groups according to the mean PNI (49.7), the 1, 3 and 5 year survival was significantly lower in the group with a low PNI (87.4\%, $72.9 \%$ and $67.7 \%$ compared to $98.3 \%, 93.3 \%$ and $86.5 \%$, respectively) [17]. When using a cut-off point of 46 in a population of gastric cancer patients, the PNI-low group had more advanced tumors according to TNM, and the PNI-low group had more often postoperative complications [16]. Based on these data, PNI could be a potential prognostic factor in gastrointestinal cancer patients, but it is unknown whether it is useful in the palliative setting of patients with PC.

\section{Quality of Life (QoL)}

Health-related QoL has become an increasingly accepted and necessary endpoint in clinical cancer trials [18]. A recent review suggested that QoL could be maintained during PIPAC-directed therapy and also improved in specific symptoms or functions scores [8]. However, more prospective data are needed to pinpoint the potential impact of PIPAC in specific symptoms or functions scores.

\section{Materials and methods}

The primary aim of this trial is to evaluate if PIPAC can induce major or complete response (PRGS 1 or 2) within a series of three PIPAC procedures in patients with PC from gastrointestinal, ovarian or primary peritoneal cancer. Secondary aims include monitoring of potential changes in QoL during the course of PIPAC treatment and assessment of the utility of MRI for the pre- and post-PIPAC evaluation of treatment response. A series of demographical data and additional variables (e.g. Prognostic Nutritional Index, PNI) obtained during the study will subsequently be used to create a model that predicts who may benefit from PIPAC treatment.

\section{Recruitment process}

Patients with PC from gastrointestinal, ovarian or primary peritoneal cancer are eligible for inclusion (Table 1). Prior to inclusion, the patients will be discussed at a dedicated multi-disciplinary tumor conference (MDT) in order to plan the best treatment strategy (e.g. PIPAC monotherapy or bidirectional therapy) and to rule out other standard treatment alternatives (Figure 1).

\section{Study intervention}

Included patients will be scheduled for three PIPAC procedures (PIPAC C/D or PIPAC OX) with intervals of 4-6 weeks or 6-7 weeks if bidirectional treatment is provided.

Table 1: Inclusion and exclusion criteria.

\begin{tabular}{lc}
\hline Inclusion criteria & Exclusion criteria \\
\hline - Histologically or cytologically verified gastrointestinal or ovarian & - Symptomatic small bowel obstruction (i.e. total parenteral \\
$\begin{array}{l}\text { cancer or primary peritoneal malignancy (based on tissue from the } \\
\text { primary tumor and/or its metastases) }\end{array}$ & nutrition, nasogastric tube) \\
- Ovarian cancer patients must be platinum resistant and have & Previous treatment with maximum cumulative doses of \\
completed at least one line of chemotherapy for platinum-resistant & anthracyclines and anthracenediones \\
disease & - A history of allergic reaction to platinum containing compounds or \\
- Radiological, histological or cytological evidence of PC & doxorubicin \\
- No indication for CRS and HIPEC (according to National Guidelines). & - Renal impairment, defined as glomerular filtration rate (GFR) $<40$ \\
- Performance status 0-1 & mL/min, (Cockcroft-Gault Equation) \\
- No more than one extraperitoneal metastasis & - Myocardial insufficiency, defined as NYHA class $>2$ \\
- Age $>18$ years & - Impaired liver function defined as bilirubin $\geq 1.5 \times$ UNL (upper \\
- Females must be post-menopausal & normal limit) \\
- Written informed consent & - Inadequate hematological function defined as ANC $\leq 1.5 \times 10^{9} / \mathrm{L}$ \\
& and platelets $\leq 100 \times 10^{9} / \mathrm{L}$ \\
\hline
\end{tabular}




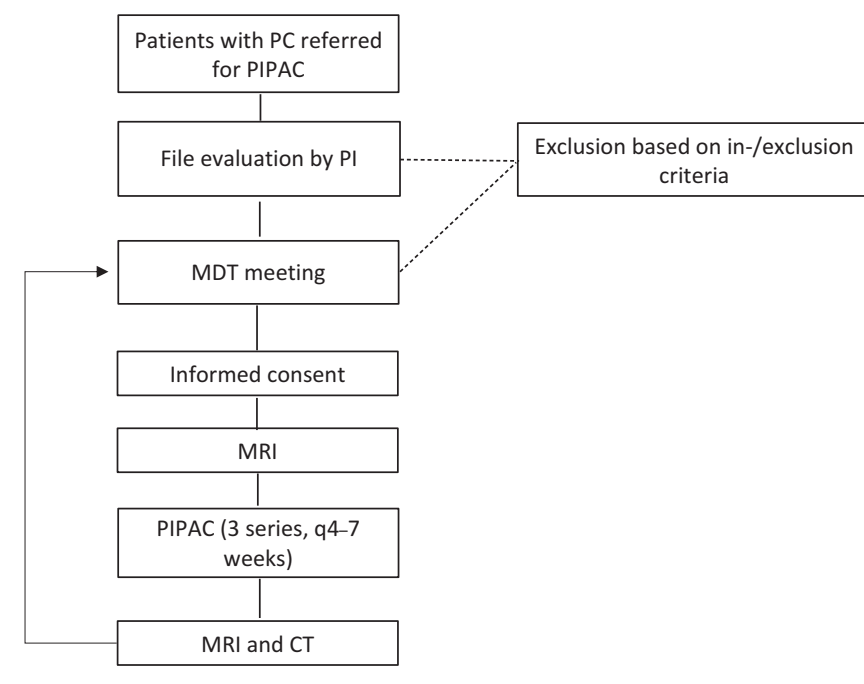

Figure 1: Patient flow chart.

MDT, multi-disciplinary tumor conference; PC, peritoneal carcinomatosis; PI, principal investigator; PIPAC, Pressurized IntraPeritoneal Aerosol Chemotherapy.

\section{MRI}

An MRI is performed at the Odense PIPAC Center (OPC) prior to the first and after three PIPAC procedures. All MRIs are performed according to a specific protocol: During the last $90-120$ min before MRI exam, the patient drinks $700-1000 \mathrm{~mL}$ of pineapple juice, and just prior to the examination, $1 \mathrm{mg}$ glucagon $\left(\right.$ GlucaGen $\left.{ }^{\circledR}\right)$ is administered subcutaneously to reduce small bowel motion. Using digital phased-array coils on an Achieva dStream 1.5T MRI system (Philips, the Netherlands), patients are examined as follows:

1. Free breathing diffusion weighted sequences with STIR Fat Sat, b-values 0, 400, 800, 1500.

2. Respiratory triggered TSE T2 sequences in two planes of upper and lower abdomen.

3. Breath hold, 3D mDixon FFE in two planes following injection of intravenous contrast (Gadovist ${ }^{\circledR}$ ).

The MRI scans will be interpreted and PCI scored by two independent experienced MR radiologists - both blinded to the laparoscopic PCI scorings.

\section{Laparoscopy, peritoneal biopsies and assessment of PRGS}

During each laparoscopy and prior to the PIPAC procedure, PCI scoring is documented and the parietal peritoneum biopsied in all four quadrants (if possible). To minimize the risk of sampling error during repeated biopsies, small metal clips are used to mark the site of biopsy. The surgeons are blinded to the results of the MRI scans. The biopsies will be fixed in formalin and embedded in paraffin, followed by step-section at three levels, which will be stained with H\&E. Microscopically, they are evaluated according to the PRGS [10]. In short, PRGS1 equals complete histological response and is defined as the absence of residual cancer and large amount of regressive fibrosis (and/or acellular mucin pools and/or infarct-like necrosis), PRGS2 is defined as a specimen where regressive changes are predominant over cancer cells, PRGS3 as a specimen where regressive changes are present but where cancer cells are predominant over fibrosis, and PRGS4 as a specimen without histological features of regression. In addition to the recommendation just mentioned, one section will be stained immunohistochemically for the tumor cell marker EP4, followed by another three step sections stained with $\mathrm{H} \& \mathrm{E}$, as described previously (Prospective, single-center implementation and response evaluation of Pressurized Intraperitoneal Aerosol Chemotherapy (PIPAC) for peritoneal metastasis, Ther Adv Med Onc, accepted March 2018). If present, the type of necrosis will also be recorded. Infarct- or mucin-like necrosis will be regarded as indicative of regression, whereas usual necrosis will be regarded as indicative of a lack of response in the respective area, as described for colorectal liver metastases [19]. In the pathology report, a PRGS for each quadrant biopsy will be given, as well as a mean PRGS.

\section{Peritoneal lavage and cytologic analysis}

Peritoneal cytology will be assessed by analysis of evacuated ascites or fluid obtained by peritoneal lavage prior to the PIPAC procedure. At least $100 \mathrm{~mL}$ peritoneal lavage fluid will be sent to the Department of Pathology, along with the biopsies, and processed as described previously (Prospective, single-center implementation and response evaluation of PIPAC for peritoneal metastasis, Ther Adv Med Onc, accepted March 2018). In short, the fluid will be centrifuged, and smears of the sediment will be analyzed by conventional cytology (Papanicolaou and May-Giemsa Grünwald staining). Leftovers of the sediment are embedded in paraffin wax and one section will be stained with H\&E. If necessary, further sections will be cut for immunocytochemical analyses for markers such as calretinin, CDX2, carcinoembryonic antigen (CEA), cytokeratin (CK) 7, CK20, Ep-CAM, Pax8 or maspin. In the pathology report, a five-tiered score will be used: malignant cells, suspicious cells, atypical cells, no malignant cells, other.

\section{PIPAC}

All PIPAC procedures will be performed laparoscopically using two standard trocars where the insufflation of normothermic $\mathrm{CO}_{2}$ maintains an intraabdominal pressure of $12 \mathrm{mmHg}$. To reduce the risk of access lesions, a transabdominal ultrasound will be performed at the start of each procedure. Following standard prophylactic antibiotics, PIPAC is performed using doxorubicin $1.5 \mathrm{mg} / \mathrm{m}^{2}$ body surface in $50 \mathrm{~mL} \mathrm{NaCl}$ $0.9 \%$ and cisplatin at a dose of $7.5 \mathrm{mg} / \mathrm{m}^{2}$ body surface in $150 \mathrm{~mL} \mathrm{NaCl}$ $0.9 \%$ (1/10 of systemic dose). In patients with PC of colorectal origin, PIPAC is performed with oxaliplatin $92 \mathrm{mg} / \mathrm{m}^{2}$ in $150 \mathrm{~mL}$ dextrose. Chemotherapy is installed at a rate of $30 \mathrm{~mL} / \mathrm{min}$ with a maximum pressure of 200 PSI and after five minutes, the chemotherapy has been delivered, and the injector is turned off. After an additional $25 \mathrm{~min}$ of simple diffusion, the intraabdominal air saturated with chemotherapy is evacuated in a closed system through a standard trocar.

Chemotherapy used in this study is standard, commercially available antineoplastic drugs, and they are part of the general assortment of the hospital pharmacy. The used drugs are traceable through the hospital pharmacy's unique batch number and through 
a patient specific chemotherapy prescription. Dose modification is not relevant in the cisplatin plus doxorubicin group. Dose modification in the oxaliplatin group will be done according to Table 2 .

Table 2: Oxaliplatin dose modification.

\begin{tabular}{ll}
\hline $\begin{array}{l}\text { Adverse } \\
\text { reaction }\end{array}$ & Action \\
\hline $\begin{array}{l}\text { Grade } 1 \\
\text { Grade } 2\end{array}$ & $\begin{array}{l}\text { No modification } \\
\text { If the toxicity is tolerable for the patient, the planned } \\
\text { treatment will continue. If the toxicity is poorly } \\
\text { tolerated, the dose of oxaliplatin will be reduced to } \\
75 \%\end{array}$ \\
Grade 3 & $\begin{array}{l}\text { The treatment will be postponed until the toxicity is } \\
\text { below grade } 2 . \text { After this, oxaliplatin can be given in } \\
\text { a dose of } 75 \% \\
\text { Stop treatment. Treatment can be reinstituted at the } \\
\text { discretion of the responsible physician, if the } \\
\text { adverse reaction falls below Grade } 2\end{array}$ \\
\hline
\end{tabular}

\section{Post procedure monitoring}

Postoperative monitoring and treatment of nausea, vomiting and pain will be according to departmental guidelines. The patient will be discharged the same day or the first day after the PIPAC procedure, but hospitalization up to three days is considered acceptable.

Adverse medical events will be graded according to Common Terminology Criteria for Adverse Events (CTCAE, version 4.0), and surgical related complications according to the Dindo-Clavien classification. The active reporting period begins, when the patient is admitted for the first PIPAC procedure and stops 30 calendar days after the last PIPAC procedure.

Quality of life questionnaires will be collected at baseline and after 4 months using the EORTC QLQ-C30 questionnaire.

\section{Study design}

This study is a prospectively controlled Phase II, single center, onearm, open-label clinical trial for patients with histological or cytological proven PC from gastrointestinal or ovarian cancer or patients with primary peritoneal cancer.

\section{Ethical declarations}

This study is GCP monitored and has been approved by the Regional Scientific Ethical Committees for Southern Denmark (IRB S-20160100) and the Danish Medicines Agency (code no 2016083464, EudraCT number 2016-003394-18). Oral and written consent from participants are mandatory.

\section{Statistics}

Values are given as means or medians where appropriate. Categorical data will be specified with $95 \%$ confidence intervals, comparisons will be performed using non-parametric tests, all p-values are twotailed and a p-value of 0.05 will be considered statistically significant. Prognostic factors indicating survival will be analyzed in a multivariable logistic regression model. Survival will be modelled in Kaplan-Meier survival curves. Baseline MRI interrater reliability will be analyzed using Cohen's kappa statistics.

An interim analysis will be performed every 6 months. Complications leading to treatment under general anesthesia (Dindo-Clavien $\geq 3 \mathrm{~b}$ ), serious adverse events in more than $10 \%$ of the included patients or a 30 days' mortality $>15 \%$ terminates the study. Median overall survival will be calculated annually, and if it is $<6$ months, the study will be stopped.

\section{Results and discussion}

\section{Hypothesis and power calculation}

The hypothesis is that PIPAC will induce a response in PC patients, and that this may be monitored by a change in the histological response evaluation. Based on the relatively few available data on PIPAC it seems relevant to divide the PC patients into two groups according to their primary cancer disease ("Colo-rectal" and "Other"), and to use this approach for the calculation of the number of patients needed.

1. Colorectal cancer patients with PC: We expect major or complete histologic response (mean PRGS 1 or 2) in $30 \%$ after SC. After a maximum of three PIPAC series, we expect major or complete response in $50 \%$. With an estimated $10 \%$ dropout, we will include 55 patients ( $\alpha$ : 0.05, Power: 0.8).

2. Other cancer patients with PC: We expect major or complete histologic response (mean PRGS 1 or 2) in $15 \%$ after SC. After a maximum of three PIPAC series, we expect major or complete response in $30 \%$. With an estimated $10 \%$ dropout, we will include 82 patients ( $\alpha$ : 0.05, Power: 0.8).

Author contributions: All the authors have accepted responsibility for the entire content of this submitted manuscript and approved submission.

Study schedule: The trial started inclusion of patients in December 2016. The last patient is estimated to be included and treated by the end of May 2021. All data generated or analyzed during this study will be included in the published article(s).

Trial organization: The trial is organized by Odense PIPAC Center (OPC) which is a multi-disciplinary cancer group based at Odense University Hospital.

Research funding: This study was funded by Odense PIPAC Center, Odense University Hospital, 5000 Odense, Denmark. 
Employment or leadership: None declared.

Honorarium: None declared.

Competing interests: The funding organization(s) played no role in the study design; in the collection, analysis and interpretation of data; in the writing of the report; or in the decision to submit the report for publication.

\section{References}

1. Coccolini F, Gheza F, Lotti M, Virzi S, Iusco D, Ghermandi C, et al. Peritoneal carcinomatosis. Wig 2013;19:6979-94.

2. Sugarbaker PH. Intraperitoneal chemotherapy and cytoreductive surgery for the prevention and treatment of peritoneal carcinomatosis and sarcomatosis. Semin Surg Oncol 1998;14:254-61.

3. Solass W, Giger-Pabst U, Zieren J, Reymond MA. Pressurized intraperitoneal aerosol chemotherapy (PIPAC): occupational health and safety aspects. Ann Surg Oncol 2013;20:3504-11.

4. Graversen M, Pedersen P, Mortensen MB. Environmental safety during the administration of pressurized intraperitoneal aerosol chemotherapy. Pleura and Peritoneum 2016;1:203-8.

5. Blanco A, Giger-Pabst U, Solass W, Zieren J, Reymond MA. Renal and hepatic toxicities after pressurized intraperitoneal aerosol chemotherapy (PIPAC). Ann Surg Oncol 2013;20:2311-6.

6. Nadiradze G, Giger-Pabst U, Zieren J, Strumberg D, Solass W, Reymond MA. Pressurized Intraperitoneal aerosol chemotherapy (PIPAC) with low-dose cisplatin and doxorubicin in gastric peritoneal metastasis. J Gastrointestinal Surg: Off J Soc Surg Alimentary Tract 2016;20:367-73.

7. Tempfer CB, Winnekendonk G, Solass W, Horvat R, Giger-Pabst $U$, Zieren J, et al. Pressurized intraperitoneal aerosol chemotherapy in women with recurrent ovarian cancer: A phase 2 study. Gynecol Oncol 2015;137:223-8.

8. Grass F, Vuagniaux A, Teixeira-Farinha H, Lehmann K, Demartines $\mathrm{N}$, Hubner M. Systematic review of pressurized intraperitoneal aerosol chemotherapy for the treatment of advanced peritoneal carcinomatosis. Br J Surg 2017;104:669-78.

9. Nowacki M, Alyami M, Villeneuve L, Mercier F, Hubner M, Willaert W, et al. Multicenter comprehensive methodological and technical analysis of 832 pressurized intraperitoneal aerosol chemotherapy (PIPAC) interventions performed in 349 patients for peritoneal carcinomatosis treatment: an international survey study. Eur J Surg Oncol 2018. https://doi.org/10.1016/j.ejso.2018.02.014.
10. Solass W, Sempoux C, Detlefsen S, Carr NJ, Bibeau F. Peritoneal sampling and histological assessment of therapeutic response in peritoneal metastasis: proposal of the peritoneal regression grading score (PRGS). Pleura and Peritoneum 2016;1:99-107.

11. Badgwell B, Cormier JN, Krishnan S, Yao J, Staerkel GA, Lupo PJ, et al. Does neoadjuvant treatment for gastric cancer patients with positive peritoneal cytology at staging laparoscopy improve survival? Ann Surg Oncol 2008;15: 2684-91.

12. Michielsen K, Vergote I, Op De Beeck K, Amant F, Leunen K, Moerman $\mathrm{P}$, et al. Whole-body MRI with diffusion-weighted sequence for staging of patients with suspected ovarian cancer: a clinical feasibility study in comparison to CT and FDG-PET/CT. Eur Radiol 2014;24:889-901.

13. Low RN, Barone RM. Combined diffusion-weighted and gadolinium-enhanced MRI can accurately predict the peritoneal cancer index preoperatively in patients being considered for cytoreductive surgical procedures. Ann Surg Oncol 2012;19:1394-401.

14. Low RN, Barone RM, Lucero J. Comparison of MRI and CT for predicting the peritoneal cancer index $(\mathrm{PCl})$ preoperatively in patients being considered for cytoreductive surgical procedures. Ann Surg Oncol 2015;22:1708-15.

15. Sun K, Chen S, Xu J, Li G, He Y. The prognostic significance of the prognostic nutritional index in cancer: a systematic review and meta-analysis. J Cancer Res Clin Oncol 2014;140:1537-49.

16. Jiang N, Deng JY, Ding XW, Ke B, Liu N, Zhang RP, et al. Prognostic nutritional index predicts postoperative complica$\underline{\text { tions }}$ and long-term outcomes of gastric cancer. Wjg 2014;20:10537-44.

17. Nozoe T, Ninomiya M, Maeda T, Matsukuma A, Nakashima H, Ezaki T. Prognostic nutritional index: a tool to predict the bio-

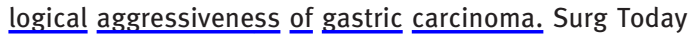
2010;40:440-3.

18. Bottomley A, Aaronson NK. International perspective on healthrelated quality-of-life research in cancer clinical trials: the european organisation for research and treatment of cancer experience. J Clin Oncol 2007;25:5082-6.

19. Chang HH, Leeper WR, Chan G, Quan D, Driman DK. Infarct-like necrosis: a distinct form of necrosis seen in colorectal carcinoma liver metastases treated with perioperative chemotherapy. Am J Surg Pathol 2012;36:570-6. 